\title{
Feedback im Arbeitsleben - eine Selbstwert-Perspektive
}

\author{
Norbert K. Semmer • Nicola Jacobshagen
}

\begin{abstract}
Zusammenfassung: Feedback ist unerlässlich und allgegenwärtig. Konstruktives Feedback ist ein wichtiges Führungsinstrument. Gutes Feedback zu geben, ist jedoch alles andere als einfach, und nicht selten fühlen sich Mitarbeitende durch Feedback verletzt und demotiviert. Ein zentraler Aspekt guten Feedbacks bezieht sich auf Aufrechterhaltung, Förderung oder Schädigung des Selbstwerts; der vorliegende Artikel beleuchtet Feedback vor allem unter diesem Aspekt. Diskutiert werden die Bedeutung des Feedbacks für den Selbstwert, Kriterien für optimales Feedback sowie die Schwierigkeiten, optimales Feedback zu geben wie auch Feedback anzunehmen, wobei negatives Feedback im Vordergrund steht. Abschließend wird auf Feedback im Rahmen betrieblicher Leistungsbewertung eingegangen.
\end{abstract}

Schlüsselwörter: Feedback $\cdot$ Selbstwert $\cdot$ Attribution $\cdot$ Wertschätzung

\section{Feedback at work: a self-esteem perspective}

\begin{abstract}
Feedback is indispensable and ubiquitary. Giving constructive feedback is an important aspect of leadership. Giving good feedback is, however, rather difficult, and one often observes that employees feel hurt and demotivated by the way feedback is given. A central aspect of good feedback relates to maintaining, enhancing, or impairing people's self-esteem, and the present article focuses on this aspect. We discuss the implications of feedback for self-esteem, criteria for optimal feedback, and the difficulties of giving and accepting feedback, especially when it is negative. The last part discusses feedback in the context of performance appraisal in organizations.
\end{abstract}

Keywords: Feedback $\cdot$ Self-Esteem $\cdot$ Attribution $\cdot$ Appreciation

\author{
Online publiziert: 11.03 .2010 \\ (C) VS-Verlag 2010 \\ Prof. Dr. N. K. Semmer $(\bowtie) \cdot$ Dr. N. Jacobshagen \\ Institut für Psychologie, Abteilung Arbeits- und Organisationspsychologie, \\ Universität Bern, Muesmattstrasse 45, \\ 3000 Bern 9, Schweiz \\ Tel.: 031-631 4045 \\ Fax: 031-631 8212 \\ E-Mail: norbert.semmer@psy.unibe.ch \\ Dr. N. Jacobshagen \\ E-Mail: nicola.jacobshagen@psy.unibe.ch
}




\section{Einleitung}

Feedback ist zunächst nicht mehr als eine Information über das Verhältnis von Ist- und Sollwert (Annett 1969; Kluger u. DeNisi 1996; Semmer u. Pfäfflin 1978). Feedback ist daher unerlässlich für eine auch nur annähernd erfolgreiche Lebensgestaltung: Wir brauchen Feedback, um zu lernen, um ungenügende oder ungewollte Ergebnisse unserer Handlungen und Entscheidungen zu korrigieren, um Gefährdungen zu vermeiden oder zu minimieren. Ohne Feedback wären wir nur schwer in der Lage, flüssig zu sprechen (wenn man auditives Feedback beim Sprechen verunmöglicht, fangen viele an zu stottern [LeeEffekt], lesbar zu schreiben (ohne visuelles Feedback verschlechtert sich die Schrift sehr schnell) oder auch nur zu gehen (weil wir ständig unsere Bewegungen and Umgebung anpassen und dabei visuelles Feedback ebenso verwerten wie propriozeptive Rückmeldungen). Feedback-Schlaufen sind daher zentraler Bestandteil vieler Forschungen und vieler theoretischer Modelle menschlichen Handelns (z. B. Hacker 2005). Dies gilt in sensumotorischer Hinsicht ebenso wie in sozialer Hinsicht, wobei es in diesem Beitrag vor allem um Letzteres geht.

So unerlässlich Feedback also ist, so problematisch ist es auch. Das hat verschiedene Aspekte, die natürlich nicht voneinander unabhängig sind: einen kognitiven, einen motivationalen und einen affektiven. Kognitive Aspekte beziehen sich darauf, dass man in der Lage sein muss, Feedback wahrzunehmen, zu interpretieren und umzusetzen. Man denke nur an Busfahrer, die so hart bremsen, dass viele Passagiere in ihrer Standfestigkeit gefährdet sind. Es ist nicht klar, ob die Fahrer gar nicht merken, dass sie so hart bremsen oder ob sie es merken, aber für normal halten, oder ob sie es gerne ändern würden, aber nicht über ausreichend feine sensorische Regulationsfähigkeiten verfügen. Motivational ist die Wahrscheinlichkeit der Zielereichung wichtig: Die Wahrnehmung, dass man sich nicht an sein Ziel annähert, oder so langsam, dass die Zielerreichung unmöglich scheint, wirkt demotivierend, während herausfordernde, aber machbar erscheinende Ziele motivierend wirken (Latham et al. 2002; Pritchard et al.1993). Die affektive Seite bezieht sich auf emotionale Reaktionen wie Stolz und Befriedigung im positiven, Frustration, Ärger, Sorgen und Verzweiflung im negativen Fall.

Es sind wohl vor allem der affektive und der (damit eng verbundene) motivationale Aspekt, der im alltäglichen (Arbeits-)Leben Probleme verursacht (Belschak u. den Hartog 2009). Mitarbeitende klagen über mangelndes Feedback (,keine Nachricht ist eine gute Nachricht"), über unfaires Feedback (,man bekommt Dinge vorgehalten, für die man gar nichts kann"), über Mangel an Respekt (Semmer et al. 2006). Andererseits sind Leistungsbeurteilungen typischerweise sehr positiv (und oft positiver, als die Führungskräfte ,privat“ denken; Levy u. Williams 2004; Murphy u. Cleveland 1995).

Umgekehrt ist positives Feedback wohl einer der wichtigsten Motivationsfaktoren (Belschak u. den Hartog 2009; Ilies u. Judge 2005; Stajkovic u. Luthans 1997; Trope u. Neter 1994). Häufig ist Feedback sogar unabhängig davon motivierend, ob es positiv oder negativ ist - es kommt oft mehr darauf an, dass es als zutreffend und fair empfunden wird (Pritchard et al. 2002). 


\section{Die Bedeutung von Feedback}

Feedback kann spezifisch auf eine Handlung oder ein Produkt bezogen sein (,auf S. 3 sind noch zwei Druckfehler"), auf generelle Handlungstendenzen oder Leistungen (,Ihre Leistung im letzten Jahr war ausserordentlich“) oder auf Personen (,Man sieht an Ihrer Art, mit den Patienten umzugehen, dass Sie Freude an Ihrem Beruf haben“). Probleme entstehen typischerweise dann, wenn das Feedback a) als auf die Person bezogen wahrgenommen wird und b) negativ ist.

Dahinter steht ein sehr grundlegendes menschliches Bedürfnis: Das Bedürfnis nach einem positiven Bild der eigenen Person, sowohl im Hinblick auf das eigene Bild von sich selbst (Selbstwert) wie auch im Hinblick auf das Bild, das andere von einem haben (Anerkennung; manche Autoren sprechen auch von sozialem Selbstwert - vgl. Semmer et al. 2006; Semmer et al. 2007; vgl. auch die Forschung zur sozialen Ausgrenzung, dem sog. Ostrazismus (Williams 2007); für eine zusammenfassende Darstellung siehe Dauenheimer et al. 2002). Dieses Bedürfnis ist auch mit typischen Wahrnehmungsverzerrungen verbunden, etwa der Tendenz, sich für besonders gut zu halten. So hält sich typischerweise eine Mehrheit von Autofahrern für überdurchschnittlich gute Autofahrer (above-averageEffekt; Dauenheimer et al. 2002), und in Organisationen halten viele Abteilungen ihren Beitrag zum Organisationsergebnis für besonders wichtig (Brown et al. 1986).

Nicht überraschend äußert sich dieses Grundbedürfnis nach einem positiven Selbstbild beim Umgang mit Feedback in vielfältiger Art und Weise. Es führt zum Beispiel dazu, dass wir negatives Feedback eher für unglaubwürdig halten - so werden Intelligenz- und Leistungstests eher für problematisch gehalten, wenn man dabei nicht gut abgeschnitten hat (Shepperd 1993). Es führt auch dazu, dass wir Feedback mehr suchen, wenn wir erwarten, dass es positiv ist, und eher vermeiden, wenn wir erwarten, dass es negativ ist (Ashford et al. 2003), dass wir Bereiche, in denen wir weniger gutes Feedback erhalten, eher für unwichtig erklären (Crocker u. Park 2003).

Natürlich gelten diese Dinge nicht im gleichen Maß für Feedback aller Art: Es kommt darauf an, wie stark das Feedback unser Ego tangiert. Einen Fehler zu machen, ist etwas anderes, als inkompetent zu gelten. So unterscheiden Kluger u. DeNisi (1996) in ihrer „Feedback-Intervention Theory“ zwischen einer Fokussierung auf das eigene Ich, die Aufgabe als Ganze, oder auf Aufgabendetails. Da Aufmerksamkeit eine begrenzte Ressource ist, führt eine Fokussierung auf das eigene Ich dazu, dass für die Aufgabe selbst weniger mentale Ressourcen zur Verfügung stehen.

Das Problem ist, dass Feedback sehr schnell personalisiert wird, also das Ego tangiert. Die Gründe dafür liegen sowohl bei den Sendenden als auch bei den Empfangenden (Schulz von Thun 2008). Nicht selten wird Feedback auf eine Art und Weise gegeben, die durch Pauschalisierungen und vorschnelle Attribution unnötig verletzt, und nicht selten wird Feedback, das man erhält, vorschnell auf die eigene Person bezogen. Feedback, vor allem negatives Feedback, gehört daher zu den schwierigsten - und oft folgenreichsten - Aspekten betrieblicher Kommunikation im Allgemeinen und des Führungshandelns im Besonderen. 


\section{Feedback geben: Worauf man achten sollte und warum es so schwierig ist}

\subsection{Gutes negatives Feedback}

Weil Feedback geben so schwierig ist, gibt es nicht wenige Ratschläge und Empfehlungen, wie man es denn geben sollte. Die Empfehlungen variieren im Detail, aber im Großen und Ganzen sind sie sich in einigen zentralen Punkten durchaus einig: Feedback sollte möglichst spezifisch sein, gut begründet sein, keine (vorschnellen) Schlüsse auf Persönlichkeit oder (unabänderliche) Fähigkeiten implizieren, nicht vorwurfsvoll sein, nicht pauschaliseren usw. (vgl. z. B. Baron 1988; London 2003; Schulz von Thun 2008).

Nur wenige dieser Punkte sind wirklich kontrovers. Darauf, genauer: auf die Frage der internalen Attribution, soll zunächst eingegangen werden. Bei anderen werden die Implikationen unseres Erachtens oft zu wenig bedacht, das gilt vor allem für die Frage der Begründung von Feedback. Und schliesslich fehlt ein wichtiger Aspekt, nämlich der der Abschwächung negativen Feedbacks durch die Bekräftigung positiver Informationen. Alle drei Punkte können unseres Erachtens von der Perspektive der Selbstwert-Bedrohung respektive -Bestätigung profitieren.

\subsubsection{Internale vs. externale Attribution}

Kontrovers diskutiert wird eigentlich nur die Frage nach der internalen Attribution. Häufig wird vorgeschlagen, alles zu vermeiden, was darauf hindeuten könnte, dass die Ursachen für einen Misserfolg in der Person selbst zu suchen sind (z. B. mangelnde Fähigkeiten, mangelnde Anstrengung - Baron 1988). Der Hintergrund für diese Auffassung ist darin zu sehen, dass internale Attribution bei Misserfolg eine Ich-Bedrohung darstellt (vgl. Ilgen u. Davis 2000), auf die eine defensive und demotivierende Reaktion zu erwarten ist. Andererseits stellt sich die Frage, ob man das eigene Verhalten ändert, wenn das Feedback impliziert, dass die Gründe außerhalb der Person zu suchen sind (etwa darin, dass zu wenig Zeit zur Verfügung stand). Ilgen u. Davis (2000) betonen daher stärker die Stabilität der Attribution: Nur wenn man davon ausgehen kann, dass Änderungen möglich sind, lohnt es sich auch, sie zu versuchen - andernfalls stellt eine Änderung ein unerreichbares Ziel dar - und unerreichbare Ziele erzeugen keine Motivation, sie zu erreichen (Latham et al. 2002; Schulz von Thun 2008).

Hier bewegt man sich also in einem Dilemma: Internale Attributionen wirken verletzend (Hareli u. Hess 2008), externale Attributionen erzeugen keine Veränderungsmotivation. Was dabei selten diskutiert wird, ist die Möglichkeit, internale Attributionen durch externe Gründe so abzuschwächen, dass das problematische Verhalten zwar der Person zugeordnet, aber zugleich nicht einfach verurteilt, sondern für verständlich und nachvollziehbar erklärt wird. Entlastende Momente können zum Beispiel sein: Die Schwierigkeit der Aufgabe (bei so komplexen Dingen können schon mal Fehler passieren; so etwas passiert anderen Leuten auch), die Erfahrung der Betroffenen (am Anfang passiert das häufig), usw. Der Vorteil dieses Vorgehens liegt darin, dass die potentiell vorwurfsvollen Elemente abgeschwächt werden, dass zugleich aber deutlich wird, dass diese entlastenden Momente nicht unbegrenzt gelten (nach dem Motto: Fehler machen ist normal und erlaubt, aber man sollte dieselben Fehler nicht wiederholt machen). 


\subsubsection{Das Problem der guten Begründung: Die Gefahr des Overkills}

Die Forderung, Feedback gut zu begründen, leuchtet auf den ersten Blick unmittelbar ein. Schließlich will man wissen, warum etwas als nicht optimal eingestuft wurde (für positives Feedback halten wir die Begründung meist nicht für so notwendig), und man will wissen, was man das nächste Mal anders machen kann. Begründungen sind also gut und notwendig. Sie zeigen, dass man sich mit dem Problem auseinandergesetzt hat und nicht einfach auf Grund eines oberflächlichen Eindrucks urteilt - oder gar auf Grund von Vorurteilen gegenüber den betroffenen Personen, auf Grund von Sympathie oder Antipathie usw. Sie sind somit ein Zeichen prozeduraler Gerechtigkeit (Cropanzano et al. 2001; Greenberg u. Colquitt 2005), und sie helfen weiter, weil sie aufzeigen, wo man etwas ändern soll.

Unter dem Aspekt der Selbst-Bedrohung kann man hier jedoch leicht zu viel des Guten tun. Einige zentrale Punkte zu benennen, macht das Feedback nachvollziehbar. Immer weitere Punkte aufzuführen oder die potentiellen Folgen auszumalen (,Wenn das Produkt so verschickt worden wäre, hätte das wahrscheinlich dazu geführt, ...") kann hingegen eine Art „Overkill“ bedeuten. Der Grund ist darin zu sehen, dass mit jedem zusätzlichen Argument, mit jeder zusätzlichen potentiellen Konsequenz und mit jeder Wiederholung immer weniger nachvollziehbar wird, warum der Fehler überhaupt je passieren konnte - schließlich ist der Mangel doch so offensichtlich, dass man es eigentlich sofort hätte bemerken müssen. Potentiell entlastende Momente werden dadurch entwertet, die Nachvollziehbarkeit des Fehlers wird immer geringer.

Natürlich muss begründet und erläutert werden, was dem Gegenüber nicht nachvollziehbar erscheint. Nicht selten aber ist nach kurzen Erläuterungen klar, wo das Problem liegt. Ab diesem Punkt werden weitere Erläuterungen, weitere Differenzierungen, das weitere Ausmalen potentieller Folgen und Wiederholungen leicht als „Nachtreten“ empfunden, mit der oben beschriebenen Wirkung. Wir haben diese Überlegungen in einem Experiment getestet, in dem Studierende das Feedback eines Dozenten beurteilen sollten. Wie erwartet, führte der „Overkill“ zu negativeren Beurteilungen als ein Feedback, das inhaltlich genauso klar war, aber zurückhaltender im Ausschmücken der negativen Aspekte (Jacobshagen et al. in Vorb.).

Aus diesen Überlegungen lässt sich ableiten, dass man Begründungen bis zu dem Punkt geben sollte, an dem das Gegenüber nachvollziehen kann, worauf man hinaus will - und nur bis zu diesem Punkt. Man könnte das als das Prinzip des „,minimal invasiven negativen Feedbacks" bezeichnen.

Es gibt noch weitere Möglichkeiten, Feedback „subtil abwertend“ zu gestalten. Dazu gehört etwa das Aufbauschen von Details (z.B. Kommafehler) oder das „Banalisieren“, indem man betont, wie einfach ein Problem zu vermeiden gewesen wäre („Sie hätten nur..."; vgl. Jacobshagen et al., in Vorb.). Wir haben hier den Overkill etwas ausführlicher beschrieben, weil dieser unter Umständen gerade aus dem Bemühen resultieren kann, die Feedback-Regel der guten Begründung umzusetzen. 


\subsubsection{Die Mischung von negativem und positivem Feedback}

Der entscheidende Punkt bei negativem Feedback ist nicht, ob man einen Fehler begangen hat. Der entscheidende Punkt liegt in den Implikationen für das eigene Selbst: Was sagt der Fehler über die eigenen Kompetenzen, die eigene Integrität, die eigene Zuverlässigkeit usw. aus? Und was sagt er über die Wertschätzung durch andere aus? Viele Feedback-Regeln, und auch die oben beschriebenen Abschwächungen, haben denn auch zum Ziel, das Feedback so wenig diagnostisch für zentrale Bereiche der Selbst- und Fremdeinschätzung werden zu lassen wie möglich. Die bis anhin dargestellten Aspekte beziehen sich dabei auf das negative Feedback im engeren Sinn. Daneben ist der Kontext dieses Feedbacks zu berücksichtigen: Welche Botschaften werden, neben dem eigentlichen negativen Feedback, noch vermittelt? Zwei Aspekte scheinen uns hier wesentlich: Zum ersten ist es wichtig, dass neben dem negativen Feedback mögliche positive Aspekte nicht aus dem Blickfeld geraten. Zum zweiten ist es wichtig, über das gerade anstehende Thema hinaus Stärken der betroffenen Person zu würdigen, was auf vielfältige Art geschehen kann.

Der erste Punkt betrifft die Kombination von negativem und positivem Feedback. Je mehr das negative Feedback in das Zentrum der Betrachtung rückt, desto mehr dominiert es den Gesamteindruck. Einen Bericht zurückzuschicken mit der einzigen Bemerkung „Dieser Bericht ist voller Tippfehler!“ lässt den gesamten Bericht als unzureichend erscheinen, auch wenn er inhaltlich für gut befunden wurde. In diesem Fall ist es wichtig, zunächst einmal die positiven Aspekte des Berichts zu betonen (Semmer u. Pfäfflin 1978; siehe auch Belschak u. den Hartog 2009).

Allerdings beinhaltet dieses Vorgehen auch Gefahren. Zum einen kann es zum Ritual werden, bei dem man während der positiven Aspekte eigentlich nur auf das ABER wartet. Zum anderen kann sich während einer Diskussion sich der Fokus unmerklich verschieben. Das Positive wird ja meist diskussionslos entgegengenommen, beim Negativen kann es schon eher passieren, dass man genauer nachfragt oder sich wehrt. Dies führt dazu, dass das Negative ausführlicher begründet, unter Umständen mehrfach wiederholt und gegen rechtfertigende Argumente verteidigt wird. Schließlich wird nur noch über das Negative gesprochen, das Positive verblasst. Deshalb muss man das Positive unter Umständen mehrfach hervorheben und so die Ich-Bedrohung abschwächen, etwa durch Bemerkungen wie „Trotz meiner Kritikpunkte will ich betonen, dass ich den Bericht insgesamt für gut/für einen hervorragenden ersten Entwurf/für eine gute Ausgangsbasis o.ä. halte.“ Dies muss man unter Umständen mehrfach tun, um der Gefahr der Fokussierung auf das Negative entgegenzuwirken.

Zum zweiten zeigt die Forschung, dass Angriffe auf das Selbst durch die Fokussierung auf Stärken abgemildert werden können und dass die Stärken und Schwächen inhaltlich nicht in einem Zusammenhang stehen müssen (Trope u. Neter 1994; Tesser 2001). Das bedeutet, dass man positive Aspekte aus allen Bereichen erwähnen kann, um die Bedrohung durch negatives Feedback abzumildern. Man kann andere Stärken erwähnen (,Ich höre nur Gutes über Ihre Zuverlässigkeit"), man kann betonen, dass man gerne mit einer Person zusammenarbeitet, man kann frühere erfolgreiche Leistungen erwähnen, usw. Und auch hier gilt: Das muss man unter Umständen mehrfach, um der Fokussierung auf das Negative entgegen zu wirken. 
Man könnte aus dem Gesagten die Konsequenz ziehen: Positives erwähnen, Negatives diskutieren und zwischendurch (wenn die Fokussierung auf das Negative zu stark zu werden droht) sowie (und das immer) am Schluss wieder Positives erwähnen. Man könnte das als „Sandwich-Feedback“ bezeichnen, das heißt, die schlechte Nachricht wird in Positives eingebettet. Unnötig zu sagen, dass das natürlich kaum funktioniert, wenn es nicht ernst gemeint ist. Und wenn man schematisch irgendetwas Positives hervorhebt, das vergleichsweise unwichtig ist („Der Bericht ist nicht gut, aber er ist schön geschrieben“), dann läuft man Gefahr, die Situation eher zu verschlechtern als zu verbessern.

\subsection{Fazit}

Als Fazit der hier dargestellten Überlegungen lässt sich sagen, dass die häufig dargestellten Feedback-Regeln der Ergänzung, teilweise auch der Korrektur bedürfen. Der entscheidende Gesichtspunkt ist: Maximale Schonung des Selbstwerts, aber zugleich Klarheit bezüglich des intendierten Inhalts. Das erfordert gute, aber nicht übertrieben ausführliche Begründungen, ein Minimum an internaler Attribution und die Einbettung in Positives, und zwar aus den verschiedensten Bereichen.

Das ist leicht gesagt, aber - wie wir alle aus unserem Alltag wissen - nicht leicht umzusetzen. Im Folgenden sollen drei Aspekte diskutiert werden, die es besonders schwer machen, gutes negatives Feedback zu geben. Der erste Aspekt betrifft Feedback, das sich nicht auf einmalige Ereignisse bezieht, der zweite betrifft erschwerende Faktoren in der Feedback gebenden, der dritte in der Feedback empfangenden Person.

\section{Warum es so schwierig ist, gutes (negatives) Feedback zu geben}

\subsection{Wenn Pauschalisierungen unvermeidlich sind}

Pauschalisierungen zu vermeiden, ist Bestandteil vieler Feedback-Empfehlungen. Impliziert ist damit die Betonung auf spezifischen Ereignissen/Verhaltensweisen/Resultaten. Was weniger diskutiert wird sind Situationen, in denen es um die Häufung problematischen Verhaltens, um das Ausbleiben von Fortschritten und ähnliches geht. Wenn jemand seine Berichte (fast) immer zu spät abliefert, wenn vergleichbare Mängel wiederholt auftreten, wenn jemand wiederholt Kolleginnen und Kollegen unfair behandelt, mit KundInnen unfreundlich umgeht usw., dann ist irgendwann eine gewisse Art von Pauschalisierung unvermeidlich. Übertrieben spezifisches Feedback hilft hier nicht weiter: „Sie haben sich am 11. Januar, am 13. Februar, am 4. März und am 21 April gegenüber $\mathrm{X}$ beleidigend verhalten“ erweckt eher den Eindruck, man sammle „Munition“ gegen die Person als dass es die Glaubwürdigkeit erhöht. Spezifik ist unerlässlich bezüglich der Art des Problems: Man sollte im obigen Beispiel die spezifischen Merkmale des unhöflichen Verhaltens möglichst gut beschreiben können. Eine gewisse Pauschalisierung bezüglich der Auftretenshäufigkeit ist jedoch unumgänglich („Ich habe jetzt wiederholt beobachtet..."). Weitere Pauschalisierungen sollten aber vermieden werden (,Sie sind offenbar ein unhöflicher Mensch“), ebenso wie Attributionen stabil-internaler Art (,Sie haben 
offenbar Spaß daran, andere fertig zu machen“). Diese Aspekte der Pauschalisierung auseinander zu halten, ist nicht ganz einfach.

Ebenso schwierig ist die Frage, wie man sich verhält, wenn man frühzeitig einen Eindruck hat, den man noch nicht verifizieren kann. Da man ihn nicht gut belegen kann: Soll man ihn verschweigen, weil man sonst jemanden ohne ausreichende Evidenz beschuldigt? Das scheint nahe zu liegen, hat aber umgekehrt den Nachteil, dass man die Person ,gegen die Wand laufen“ lässt, wenn man etwas frühzeitig bemerkt und es nicht sagt. Unseres Erachtens sind das Situationen, in denen Ich-Botschaften besonders wichtig sind. „Ich habe den Eindruck, dass Sie sich bei dieser Art Aufgaben nicht besonders wohl fühlen“" informiert über den Eindruck, ohne ein definitives Urteil nahe zu legen.

Und schließlich finden sich kaum Hinweise darauf, wie man mit Situationen umgeht, in denen man tatsächlich zu dem Schluss kommt, dass jemand für bestimmte Aufgaben/ Positionen und ähnliches nicht geeignet ist: Zum Beispiel, wenn man junge MusikerInnen unterrichtet und den Eindruck hat, jemand sei nicht gut genug für eine Profi-Karriere oder wenn man jemandem die Leitung einer Arbeitsgruppe oder einer Abteilung nicht zutraut usw. Hier stellt also gerade das Merkmal den Kern des Feedbacks dar, das typischerweise beim Feedback gerade vermieden werden soll, nämlich die stabil-internale Attribution. Auch hier liegt unseres Erachtens der Schlüssel darin, jeder Pauschalisierung auf die Kompetenzen der Person insgesamt entgegen zu wirken (,Sie sind ein hervorragender Chemiker! Die Leitung des Labors erfordert aber viel mehr als die hohe Kompetenz als Chemiker!"). Wie definitiv man dabei vorgeht, ist natürlich eine Frage der jeweiligen Aufgaben: Man kann jemandem die Leitung einer Arbeitsgruppe übertragen, nachdem man gewarnt hat, dass man skeptisch sei, aber eine Chance geben wolle. Dasselbe ist bei der Ernennung zum Laborleiter wohl nicht mehr sinnvoll.

Allerdings ist immer zu prüfen, ob Schwächen kompensierbar sind. Dies kann einerseits in der Person erfolgen (Ausbildungsmaßnahmen), andererseits auch im größeren System. Nicht jede Führungskraft ist beispielsweise gut darin, sowohl aufgabenorientiertes als auch mitarbeiterorientiertes Verhalten zu zeigen (Rosenstiel 2000). Wenn sie sich darüber im Klaren ist, kann man versuchen, individuelle Schwächen durch einen systemischen Ausgleich zu kompensieren - etwa, indem eine stark aufgabenorientierte Leiterin einen stark mitarbeiterorientierten Stellvertreter erhält.

Zudem muss immer geprüft werden, ob Dinge nicht deshalb anhaltende Probleme darstellen, weil die Person ,nun einmal so ist“, sondern deshalb, weil die Person unter anhaltenden schwierigen Umständen lebt. Eine allein erziehende Mutter wird mehr Probleme haben, immer pünktlich zu sein, und dann sollte man prüfen, ob man sie unterstützen kann, zum Beispiel durch Flexibilisierung der Arbeitszeit.

Grundsätzlich muss man aber trotzdem anerkennen, dass Menschen Stärken und Schwächen haben, die nicht ohne weiteres änderbar sind. Man muss sorgfältig prüfen, ob man da nicht voreilige Schlüsse zieht (im Sinne des fundamentalen Attributionsfehlers - Fincham u. Hewstone 2003) und ob es andere Lösungsmöglichkeiten gibt. Aber diese Prüfung kann auch dazu führen, dass eine stabil-internale Attribution tatsächlich angemessen ist.

Noch ein weiterer Gesichtspunkt kommt hinzu: So sehr man den Selbstwert einer Person schonen, ihr allenfalls Entwicklungsmöglichkeiten zubilligen will, so sehr muss man auch berücksichtigen, inwieweit andere Personen davon betroffen sind. Wer - durch man- 
gelnde Leistung, durch rüdes Verhalten, durch Unzuverlässigkeit usw. - anderen Schaden zufügt, muss relativ schnell klares Feedback erhalten und lernen, dass das nicht toleriert wird.

Die soziale Einbettung individuellen Feedbacks müsste unseres Erachtens ganz allgemein mehr gewichtet werden. So kann positives Feedback gegenüber einer Person zu negativen Reaktionen bei anderen führen, weil die den Eindruck haben, der/die andere erhalte positiveres Feedback als sie selbst, werde vom Chef/der Chefin“ gehätschelt, u. ä.

\subsection{Probleme beim Sender}

Mangelnde Feedback-Kultur wird häufig beklagt. In einer Tagebuchstudie von EillesMatthiessen u. Zapf (2000) berichteten die Teilnehmer, mindestens einmal im Monat eine Situation zu erleben, in denen sie sich von ihren Vorgesetzten gedemütigt fühlten; dabei spielte Feedback keine unwesentliche Rolle. Auch in unseren Konsultationen und in vielen Interviews taucht als problematisch empfundenes Feedback regelmäßig auf. Sieht man vorläufig davon ab, dass dabei auch Empfindlichkeiten der Feedback-Empfänger mitspielen können, so stellt sich die Frage, warum eigentlich problematisches Feedback immer wieder auftritt - und zwar keineswegs nur von Vorgesetzten gegenüber Mitarbeitenden, sondern auch im Umgang von Mitarbeitenden untereinander.

Ein Grund für inadäquates Feedback ist sicherlich mangelnde soziale Kompetenz. Oft weiß man zwar im Allgemeinen, wie man Feedback geben sollte, verfügt aber nicht in ausreichendem Masse über die Kompetenz, subtile Aspekte (z. B. das oben dargestellte „Overkill“) wahrzunehmen. Verbunden damit ist häufig mangelnde Empathie: Man hat Mühe nachzuvollziehen, warum andere Mühe haben oder Fehler machen. Dann neigt man schnell dazu, Ursachen für problematisches Verhalten oder mangelnde Leistung auf stabil-internale Ursachen zu attribuieren (fundamentaler Attributionsfehler). Zudem unterliegt man dem sog. „Hindsight-Bias“, das heißt, man geht zu schnell davon aus, dass unerwünschte Ereignisse oder Resultate für die Betroffenen gut vorhersehbar waren (vgl. Reason 2008).

Diese Tendenzen werden unter Umständen durch Reaktionstendenzen unterstützt, die wir alle bis zu einem gewissen Grad aufweisen, die aber bei manchen Menschen etwas stärker ausgeprägt sind. Dazu gehören zum Beispiel die Tendenz, Erfolge sich selbst, Misserfolge eher den Umständen oder anderen Personen zuzuschreiben oder die Tendenz, andere abzuwerten, wenn es dem eigenen Selbstwert dient (Leary 2007). Auch mangelnde Selbstkontrolle ist hier zu nennen, was dazu führen kann, dass man den eigenen Emotionen (in diesem Fall typischerweise Ärger) freien Lauf lässt und damit härter Feedback gibt, als es angemessen ist - und oft auch härter, als man selbst möchte, wenn man die Angelegenheit im Nachhinein neu bewertet (vgl. Baron 1988).

Feedback, das aus unserer Sicht problematisch ist, kann aber auch gewollt sein, weil man den Mitarbeitenden misstraut und davon ausgeht, dass nur Kontrolle und Härte die Menschen motivieren (vgl. McGregor's Theorie X und Y - Greif 1992). Auch Aspekte der Selbstdarstellung (man möchte als harter Kerl gelten) können hier eine Rolle spielen.

Und schließlich gibt es auch die andere Seite: Man hat Angst, jemanden zu verletzen, Konflikte zu provozieren, demotivierend statt motivierend zu wirken, usw. - und 
vermeidet negatives Feedback. Zum Teil wird es gar nicht geäußert (ein Grund, warum Mitarbeiterbeurteilungen oft so positiv sind), zum Teil wird es hinausgeschoben, was die Gefahr beinhaltet, dass es dann umso massiver gegeben wird (Larson 1989) und umso heftigere Reaktionen provoziert - eingeschlossen dem Vorwurf, dass dieses Feedback so spät komme.

Mangelnde Bereitschaft (oder mangelnder Mut) negatives Feedback zu geben, ist ein verbreitetes Problem. Nicht zuletzt für Führungskräfte kann es zur gefährlichen Falle werden, denn die Tendenz, Vorgesetzte direkt zu kritisieren, ist nicht sehr ausgeprägt (Tourish u. Robson 2006). Vorgesetzte laufen daher Gefahr, von kritischem Feedback abgeschirmt zu werden (und sie verstärken diese Gefahr manchmal, in dem sie defensiv auf Feedback reagieren - s. u.).

Ein letzter Punkt in Bezug auf das Geben von Feedback betrifft unbeabsichtigtes Feedback. Wenn eine Führungskraft beim Betriebsfest an einem Tisch länger sitzt als an anderen, kann das schnell dazu führen, dass ihr Verhalten als intentional angesehen und als unfair beurteilt wird (,Sie sitzt wieder mal bei ihren Lieblingen“), auch wenn die längere Verweildauer damit gar nichts zu tun hat, sondern zum Beispiel auf eine besonders interessante Diskussion zurückzuführen ist. Führungskräfte müssen damit rechnen, dass jeder ihrer Verhaltensweisen Absicht unterstellt wird. Wenn man jemandem begegnet und nicht grüßt, weil man ganz in Gedanken ist; wenn man eine Abteilung schon länger nicht mehr besucht hat, weil an dem Tag, an dem man kommen wollte, etwas dazwischen kam - für Dinge dieser Art wird, wie generell im zwischenmenschlichen Umgang, schnell unterstellt, das sei Absicht. Das stellt hohe, fast unerfüllbare Anforderungen an Führungskräfte (und ist vielleicht mit dafür verantwortlich, dass sie oft eher Kontakt mit ihresgleichen suchen als mit Mitarbeitenden, weil dort diese Art Beobachtung weniger auftritt).

Diesen Absatz abschließend sei noch betont, dass natürlich nie nur einzelne Verhaltensweisen zählen, sondern letztlich die vorherrschende Kultur ausschlaggebend ist. Wer als Führungskraft akzeptiert ist, hat auch einen gewissen ,idiosynkratischen Kredit“; da werden solche Dinge weniger schnell im Sinne finsterer Absichten interpretiert (vgl. auch Ullrich et al. 2009).

\subsection{Probleme beim Empfänger: Feedback suchen und annehmen}

Man darf sich aber nicht der Illusion hingeben, wenn man ,optimales“ Feedback gebe, sei alles in Ordnung. Feedback ist immer eine Angelegenheit mehrerer Parteien. Und so, wie die Feedback-Gebenden nicht selten Fehler machen, so muss man auch damit rechnen, dass diejenigen, die Feedback erhalten, darauf mit übertriebener Empfindlichkeit reagieren. Verschiedene Persönlichkeitsmerkmale können zu solcher Überempfindlichkeit beitragen (Semmer u. Meier 2009). Dazu gehört geringe emotionale Stabilität (Bernichon et al. 2003), dazu kann ein geringer Selbstwert gehören, dazu gehört aber vor allem ein hoher, aber instabiler Selbstwert. Wer einen hohen, aber fragilen Selbstwert hat, neigt dazu, auf empfundene Ungerechtigkeit besonders heftig zu reagieren (Meier et al. 2009; Semmer u. Meier 2009). Ein fragil-hoher Selbstwert ist nicht zuletzt typisch für Personen mit narzisstischen Tendenzen (Meier u. Semmer 2008).

Auch die Zielorientierung spielt eine wichtige Rolle. Eine wichtige Unterscheidung betrifft den sogenannten Regulations-Focus (regulatory focus), der eher promotions- 
orientiert oder eher präventionsorientiert sein kann (Shah u. Higgins 1997). Promotionsorientierung ist durch das Anstreben herausfordernder Ziele gekennzeichnet: Werden sie erreicht, empfindet man Stolz und Freude, werden sie verfehlt, empfindet man Enttäuschung. Präventionsorientierung hingegen ist durch Vermeidungsziele gekennzeichnet: Man will Negatives (z. B. eine Niederlage, eine Blamage) verhindern; werden diese Ziele erreicht, ist man beruhigt, werden sie verfehlt, ist man nervös und angespannt. Präventions- und Promotionsorientierung spiegeln einerseits Merkmale der Person wider. Sie kann aber auch durch die Situation gefördert werden, etwa durch die Art, wie Vorgesetzte oder Teams ihre Ziele oder eine Aufgabenstellung definieren - so gelten Überwachungsaufgaben eher als präventionsorientiert, kreative Aufgaben und Aufgaben, die erfordern, dass man Risiken eingeht, als promotionsorientiert (Van-Dijk u. Kluger 2004). In Bezug auf Feedback lässt sich sagen, dass bei Promotionsorientierung positives Feedback besonders motivierend sein kann, bei Präventionsorientierung hingegen negatives Feedback (Van-Dijk u. Kluger 2004). Das heißt nicht, dass man Präventionsorientierten ständig negatives Feedback geben sollte: Dauerhaft negatives Feedback ist mit Sicherheit nicht motivationsfördernd. Es kann aber zum Beispiel heißen, dass man - in Bezug auf eine bestimmte Aufgabe - das Glas eher als halb leer darstellt (wir sind noch nicht so weit) oder die negativen Folgen einer Zielverfehlung betont (wenn wir das nicht schaffen, verlieren wir den Auftrag), während man bei Promotionsorientierten eher betont, dass man doch große Fortschritte mache und das Ziel in Reichweite sei, und dass Zielerreichung mit großartige Chancen mit sich bringe. Diese Art Feedback muss auf eine bestimmte Aufgabe oder eine bestimmte Situation bezogen sein, und alle übrigen Feedback-Regeln, so etwa die Kombination mit positivem Feedback, gelten selbstverständlich weiter. Die hier erwähnten Forschungsergebnisse sind also nicht als „Freibrief“ für undifferenziertes negatives Feedback bei Vorherrschen von Präventionsorientierung zu verstehen.

Noch eine weitere Art von Zielorientierung ist wichtig. Man kann stärker aufgabenbezogene Ziele (task-goals; oft auch als learning-goals oder als mastery-goals bezeichnet) oder stärker Ich-bezogene Ziele haben (ego-goals, auch performance-goals oder abilitygoals). Generell zeigt sich, dass aufgabenbezogene Ziele dazu führen, dass man Feedback vor allem im Hinblick auf den eigenen Lernfortschritt bewertet, und dass negatives Feedback zu erhöhten Anstrengungen führt. Negatives Feedback wird dann als nützlich für den eigenen Fortschritt angesehen, und diese ,instrumentelle“ Motivation verstärkt die eigene, aktive Suche nach Feedback (Ashford et al. 2003). Ego-Ziele sind häufig damit verbunden, dass man Feedback im Hinblick auf den sozialen Vergleich (,,bin ich besser als andere?“) oder im Hinblick auf eigene Fähigkeiten (,bin ich intelligent?") bewertet und sucht; negatives Feedback führt vor allem bei einem Fähigkeitsfokus häufig zu Motivationsverlust (vgl. für einen Überblick Grant u. Dweck 2003). Wenn ich-bezogene Aspekte im Vordergrund stehen, wird negatives Feedback seltener gesucht, eher abgewertet und eher verzerrt (Ashford et al. 2003).

Wir haben weiter oben schon darauf hingewiesen, dass Führungskräfte oft zu wenig negatives Feedback erhalten. Führungskräfte müssten daher besonders aktiv nach negativem Feedback suchen und besonders wenig defensiv reagieren, wenn sie es erhalten. Leider ist nicht selten das Gegenteil der Fall (Ashford et al. 2003). Dazu mag beitragen, dass Führungskräfte oft Entscheidungen treffen müssen, die irgendjemandem nicht gefallen oder dass sie ihre Pläne oft gegen Widerstand und Bedenken durchsetzen müssen. 
Dies kann aber dazu führen, dass man Kritiker generell als „Bedenkenträger“ abstempelt, und dass man - bestärkt durch das überwiegend positive Feedback - immer mehr zu der Überzeugung kommt, Recht zu haben. Das kann leicht dazu führen, dass man Kritiker spüren lässt, dass ihre Kritik unerwünscht ist - mit der Folge, dass man immer mehr von Leuten umgeben ist, die einem schmeicheln. Man könnte diese Entwicklung als „Narzissmus-Spirale“ bezeichnen. Dies kann auch das Phänomen des „Groupthink“ fördern, bei dem abweichende Meinungen bestraft werden und eine Illusion der Unverletzlichkeit vorherrscht (Esser 1998).

Im Allgemeinen suchen Menschen aktiv nach Feedback. Dies kann einerseits dadurch geschehen, dass man explizit Feedback einfordert. Dies ist im Allgemeinen mit besserer Leistung verbunden; darüber hinaus ist die aktive Suche nach negativem Feedback mit positiveren Einschätzungen durch Vorgesetzte verbunden, zu deutliches Interesse in positives Feedback hingegen mit negativeren (Ashford et al. 2003). Neben dem expliziten Feedback kann man aber auch die Umgebung implizit nach Feedback absuchen. Das tut man z.B. häufiger, wenn man negatives Feedback erwartet. Nicht selten werden dabei relativ subtile Hinweise interpretiert, wie sie weiter oben im Hinblick auf Führungsverhalten beschrieben wurden (Ashford et al. 2003).

\section{Mitarbeiter-Beurteilung}

Ein letzter Punkt, den wir noch diskutieren wollen, bezieht sich auf Mitarbeiter-Beurteilung. Wir können an dieser Stelle nicht umfassend darauf eingehen, möchten aber einige Punkte kurz hervorheben. Bei der Mitarbeiter-Beurteilung geht es um Kriterien, nach denen Leistung und Verhalten einer Person bewertet wird; das Ziel ist die Förderung der Leistung. Die Annahme, dass Leistungsbewertung leistungssteigernd wirkt, ist auch nahe liegend, denn Feedback hat eine enorme Wirkung auf Verhalten. So zeigt Zohar (2002), dass konsequentes Ansprechen sicherheitsrelevanter Verhaltensweisen in Mitarbeitergesprächen dazu führte, dass die Zahl von Verletzungen ab- und der Gebrauch von Gehörschutz zunahm und das empfundene Sicherheitsklima anstieg. Das Productivity Measurement and Enhancement System (ProMES; deutsch: Partizipatives Produktivitätsmanagement; Pritchard et al. 1993) bewirkt enorme Produktivitätssteigerungen durch Feedback, das auf einem Messsystem beruht, das unter Beteiligung der Mitarbeitenden entwickelt wird und die Leistung der Betroffenen umfassend und fair widerspiegelt (Pritchard et al. 2008).

Dennoch ist die Auffassung, Mitarbeiterbewertung führe zu höherer Produktivität, in vielen Fällen ungerechtfertigt. Messungen und darauf basierendes Feedback ist in erster Linie ein Instrument zur Leistungs-Steuerung. Ob diese Steuerung dazu führt, dass das Richtige getan wird, ist eine ganz andere Frage, und sie muss nicht selten mit Nein beantwortet werden (vgl. Kluger u. DeNisi 1996). Der Erfolg des erwähnten ProMES-Konzepts (Pritchard et al. 2002) beruht darauf, dass das Messsystem die Leistung umfassend und fair widerspiegelt. Das erfordert aufwendige Entwicklungsarbeiten. Demgegenüber werden nicht selten Leistungsindikatoren gewählt, die leicht zu messen sind und daher a) wenig Aufwand verursachen und b) als ,objektiv“ gelten. Solche Indikatoren sind häufig einseitig (z.B. vor allem auf Quantität ausgerichtet), nicht selten durch die Betroffenen 
gar nicht ohne weiteres zu beeinflussen (etwa die Komplexität des Problems, das eine anrufende Person präsentiert), und vielfach sogar im Widerspruch zu sinnvollen Kriterien. Ein Beispiel ist die Vorgabe von Zeiten für Telefongespräche im Call Center und die Bewertung nach der Zahl der Gespräche. Das kann leicht zu Dilemmata führen, weil man ja auch gehalten ist, auf KundInnen einzugehen und ihre Probleme ernst zu nehmen. Wenn die Zahl der Gespräche für die Bewertung dominiert, kann das leicht die Qualität der Gespräche beeinträchtigen.

Ein weiteres Problem ergibt sich beim sog. $360^{\circ}$-Feedback, das vor allem bei Führungskräften eingesetzt wird. Dabei erhält man von mehereren Seiten - Vorgesetzte, Kolleginnen/Kollegen und Mitarbeitenden - Feedback. Das kann nützlich sein, vor allem, wenn verschiedene Leistungsindikatoren jeweils von denen eingeschätzt werden, werden, die sie am besten beurteilen können. Das ist nicht immer der Fall. Zudem kann $360^{\circ}$ Feedback auch widersprüchlich sein, wenn die verschiedenen Personen zu unterschiedlichen Urteilen kommen. Wichtig ist dann, dass man den Betroffenen nicht einfach das Feedback präsentiert, sondern sie bei dessen Interpretation unterstützt. Wie alle formalisierten Feedback-Systeme, bedarf auch $360^{\circ}$-Feedback der sorgfältigen Umsetzung, wenn Schaden vermieden werden soll. Insbesondere ist zu betonen, dass $360^{\circ}$ Feedback für Entwicklungs-Zwecke entwickelt wurde und nicht für betriebliche Entscheidungen wie etwa Beförderungen herangezogen werden sollte (vgl. dazu DeNisi u. Kluger 2000).

In der Regel richten die Beschäftigten ihr Verhalten danach aus, was vor allem bewertet wird ("what you measure is what you get"). Bei einseitiger Betonung einzelner Kriterien kann das schnell dazu führen, dass die Leistung im umfassenden Sinn dadurch eher beeinträchtigt als gefördert wird. So kann es passieren, dass Call-Center Agenten Gespräche abbrechen, obwohl das geschilderte Problem eigentlich mehr Zeit verlangt; dass Mitarbeiter, die nach Umsatz bezahlt werden, den KundInnen Unnötiges aufschwatzen; dass in einer Versicherung leicht überhöhte Rechnungen nicht beanstandet werden, weil Kundenreklamationen die Leistungsbewertung beeinträchtigen (Kerr 1975; siehe auch Mihalic u. Ludwig 2009).

Einseitige Bewertungssysteme führen aber nicht nur dazu, dass die organisationale Leistung beeinträchtigt statt gefördert wird. Sie führt auch leicht zu Verbitterung und Zynismus unter den Mitarbeitenden, die ihre Leistung durch dieses System nicht fair gewürdigt sehen, und die ständig vor dem Konflikt stehen, das zu tun, was sie für sinnvoll halten oder das, was von den Bewertungskriterien gewürdigt wird: Handeln sie sinnvoll, wird ihre Leistung als nicht optimal eingestuft; wird hingegen ihre Leistung als gut eingestuft, haben sie selbst das Gefühl, für das Falsche gelobt zu werden, und sie sehen, wie andere, die sich genau an die Vorgaben halten, besser dastehen als sie selbst.

Und: Auch wenn leistungsbezogene Vergütung die Leistung erhöht - Feedback und Anerkennung haben eine vergleichbare Wirkung (Stajkovic u. Luthans 1997), aber mit weniger Nebenwirkungen.

\section{Abschliessende Bemerkungen: Feedback und Unternehmenskultur}

Wir haben bereits weiter oben darauf hingewiesen, dass einzelne Feedbacks eine andere Bedeutung erhalten können, je nachdem, wie die Unternehmenskultur insgesamt aus- 
geprägt ist. Entscheidend ist eine allgemeine Kultur der Wertschätzung. Mitarbeitende müssen spüren, dass man sie schätzt, dass man sie unterstützt, dass man ihre Leistung würdigt, dass man ihre Anliegen ernst nimmt. Wertschätzung kann auf vielfältige Weise vermittelt werden. Dazu gehört natürlich das Lob, dazu gehört aber auch, andere um ihre Meinung zu fragen und diese ernst zu nehmen. Auch Arbeitsgestaltung kann Wertschätzung vermitteln: Interessante Aufgaben zu übertragen und dabei Spielräume zu gewähren, wie diese ausgeführt werden, signalisiert Vertrauen in Kompetenz und Verlässlichkeit der Betroffenen - ebenso wie eintönige Arbeiten und kleinliche Kontrollen das Gegenteil vermitteln (vgl. Semmer et al. 2006). Wertschätzung erzeugt ein Klima der ,psychologischen Sicherheit" (Edmondson 2004), in dem man nicht ständig Gefahr läuft, abgekanzelt zu werden und in dem Fehler und Probleme offen angesprochen werden können (siehe auch das Konzept der Fehlermanagement-Kultur - van-Dyk et al. 2005). Das Erleben von Wertschätzung geht mit besserem Befinden einher (Jacobshagen u. Semmer 2009; Stocker et al. im Druck); vor dem Hintergrund einer wertschätzenden Organisationskultur kann man aber wohl auch besser mit Feedback, insbesondere mit negativem Feedback, umgehen.

\section{Literatur}

Annett, J. (1969). Feedback and human behaviour: The effects of knowledge of results, incentives and reinforcement on learning and performance. Oxford: Penguin.

Ashford, S. J., Blatt, R., \& VandeWalle, D. (2003). Reflections on the looking glass: A review of research on feedback-seeking behavior in organizations. Journal of Management, 29, 773-799.

Baron, R. A. (1988). Negative effects of destructive criticism: Impact on conflict, self-efficacy, and task-performance. Journal of Applied Psychology, 73, 199-207.

Belschak, F. D., \& den Hartog, D. N. (2009). Consequences of positive and negative feedback: The impact on emotions and extra-role behaviors. Applied Psychology: An International Review, $58,274-303$.

Bernichon, T., Cook, K. E., \& Brown, J. D. (2003). Seeking self-evaluative feedback: The interactive role of global self-esteem and specific self-views. Journal of Personality and Social Psychology, 84, 194-204.

Brown, R., Condor, S., Mathews, A., Wade, G., \& Williams, J. (1986). Explaining intergroup differentiation in an industrial organization. Journal of Occupational Psychology, 59, 273-286.

Crocker, J., \& Park, L. E. (2003). Seeking self-esteem: Maintenance, enhancement, and protection of self-worth. In M. R. Leary \& J. P. Tangney (Hrsg.), Handbook of self and identity (S. 291-313). New York: Guilford Press.

Cropanzano, R., Byrne, Z. S., Bobocel, D. R., \& Rupp, D. E. (2001). Moral virtues, fairness heuristics, social entities, and other denizens of organizational justice. Journal of Vocational Behavior, 58, 164-209.

Dauenheimer, D., Stahlberg, D., Frey, D., \& Petersen, L. E. (2002). Die Theorie des Selbstwertschutzes und der Selbstwerterhöhung. In D. Frey \& M. Irle (Hrsg.), Theorien der Sozialpsychologie, Band 1: Motivations- und Informationsverarbeitungstheorien (2. Aufl., S. 159-190). Bern: Huber.

DeNisi, A. S., \& Kuger, A. N. (2000). Feedback effectiveness: Can 360-degree appraisals be improved? Academy of Management Executive, 14, 129-139. 
Edmondson, A. C. (2004). Psychological safety, trust, and learning in organizations: A group-level lens. In R. M. Kramer \& K. S. Cook, (Hrsg.), Trust and distrust in organizations: Dilemmas and approaches (S. 239-272). New York: Russell Sage Foundation.

Eilles-Matthiessen, C., \& Zapf, D. (2000). Führungskultur verträgt kein sozial inkompetentes Vorgesetztenverhalten. Personalführung, 33(12), 34-41.

Esser, J. K.(1998). Alive and well after 25 years: A review of groupthink research. Organizational Behavior and Human Decision Processes, 73, 116-141.

Fincham, F., \& Hewstone, M. (2003). Attributionstheorie und -forschung - Von den Grundlagen zur Anwendung. In W. Stroebe, K. Jonas, \& M. Hewstone (Hrsg.), Sozialpsychologie (4. Aufl., S. 215-264). Berlin: Springer.

Grant, H., \& Dweck, C. S. (2003). Clarifying achievement goals and their impact. Journal of Personality and Social Psychology, 85, 541-553.

Greenberg, J., \& Colquitt, J. A. (2005). Handbook of organizational justice. Mahwah, NJ: Lawrence Erlbaum.

Greif, S. (1992). Konzepte der Organisationspsychologie. Bern: Huber.

Hacker, W. (2005). Allgemeine Arbeitspsychologie. Bern: Huber.

Hareli, S., \& Hess, U. (2008). The role of causal attribution in hurt feelings and related social emotions elicited in reaction to other's feedback about failure. Cognition and Emotion, 22, 862-880.

Ilgen, D. R., \& Davis, C. A. (2000). Bearing bad news: Reactions to negative performance feedback. Applied Psychology: An International Review, 49, 550-565.

Ilies, R., \& Judge, T. A. (2005). Goal regulation across time: The effects of feedback and affect. Journal of Applied Psychology, 90, 453-467.

Jacobshagen, N. \& Semmer, N. K. (2009). Wer schätzt eigentlich wen? Kunden als Quelle der Wertschätzung am Arbeitsplatz. Wirtschaftspsychologie, 11, 11-19.

Jacobshagen, N., Semmer, N. K., Müller, H., Zumkemi, M., \& Elfering, A. (in Vorb.) Subtly derogating feedback: Its characteristics and its effect on the acceptance of negative feedback. Bern: Universität Bern, Institut für Psychologie.

Kerr, S. (1975). On the folly of rewarding A, while hoping for B. Academy of Management Journal, 18, 769-783. (Reprint in: R. P. Vecchio (Hrsg.). (2007), Leadership: Understanding the dynamics of power and influence in organizations (2. Aufl., S. 228-238). Notre Dame, IN, US: University of Notre Dame Press.

Kluger, A. N. \& DeNisi, A. (1996). The effects of feedback interventions on performance: A historical review, a meta-analysis, and a preliminary feedback intervention theory. Psychological Bulletin, 119, 254-284.

Larson, J. R. (1989). The dynamic interplay between employees' feedback-seeking strategies and supervisor's delivery of performance feedback. Academy of Management Review, 14, 408-422.

Latham, G. P., Locke, E. A., \& Fassina, N. E. (2002). The high performance cycle: Standing the test of time. In S. Sonnentag (Hrsg.), The psychological management of individual performance: A handbook in the psychology of management in organizations (S. 201-228). Chichester, UK: Wiley.

Leary, M. R. (2007). Motivational and emotional aspects of the self. Annual Review of Psychology, $58,317-344$.

Levy, P. E., \& Williams, J. R. (2004). The social context of performance appraisal: A review and framework for the future. Journal of Management, 30, 881-905.

London, M. (2003). Job feedback: Giving, seeking, and using feedback for performance improvement (2. Auff.). Mahwah, NJ: Lawrence Erlbaum.

Meier, L. L., \& Semmer, N. K. (2008). When do narcissists get stressed? The role of the imbalance between effort and reward. Manuscript submitted for publication. 
Meier, L. L., Semmer, N. K., \& Hupfeld, J. (2009). The impact of unfair treatment on depressive mood: The moderating role of self-esteem level and self-esteem instability. Personality and Social Psychology Bulletin, 35, 643-655.

Mihalic, M. T., \& Ludwig, T. D. (2009). Behavioral system feedback measurement failure: Sweeping quality under the rug. Journal of Organizational Behavior Management, 29, 155-174.

Murphy, K. R., \& Cleveland, J. N. (1995). Understanding performance appraisal: Social, organizational, and goal-based perspectives. Thousand Oaks: Sage.

Pritchard, R. D., Kleinbeck, U. \& Schmidt, K.-H. (1993). Das Managementsystem PPM. Durch Mitarbeiterbeteiligung zu höherer Produktivität. München: Beck.

Pritchard, R. D., Harrell, M. M., Diaz Granados, D., \& Guzman, M. J. (2008). The Productivity Measurement and Enhancement System: A meta-analysis. Journal of Applied Psychology, 93, 540-567.

Pritchard, R. D., Holling, H., Lammers, F., \& Clark, B. D. (Hrsg.). (2002). Improving organizational performance with the Productivity Measurement and Enhancement System: An international collaboration. New York: Nova Science.

Reason, J. T. (2008). The human contribution: Unsafe acts, accidents and heroic recoveries. Farnham: Ashgate.

Rosenstiel von, L. (2000). Grundlagen der Organisationspsychologie (4. Aufl.). Stuttgart: Schäffer-Poeschel Verlag.

Schulz von Thun, F. (2008). Miteinander reden: Störungen und Klärungen (46. Aufl.). Reinbek bei Hamburg: Rowohlt Taschenbuch Verlag.

Semmer, N. \& Pfäfflin, M. (1978). Interaktionstraining. Ein handlungstheoretischer Ansatz zum Training sozialer Fertigkeiten. Weinheim: Beltz.

Semmer, N. K., \& Meier, L. L. (2009). Individual differences, work stress, and health. In J. Quick, M. J. Schabracq, \& C. L. Cooper (Hrsg.), Handbook of work and health psychology (3. Aufl., S. 99-121). Chichester, UK: Wiley.

Semmer, N. K., Jacobshagen, N., \& Meier, L. L. (2006). Arbeit und (mangelnde) Wertschätzung. Wirtschaftspsychologie, 8, 87-95.

Semmer, N. K., Jacobshagen, N., Meier, L. L., \& Elfering, A. (2007). Occupational stress research: The „Stress-as-Offense-to-Self“ perspective. In J. Houdmont \& S. McIntyre, (Hrsg.), Occupational health psychology: European perspectives on research, education and practice, Vol. 2 (S. 43-60). Castelo da Maia, Portugal: ISMAI Publishing.

Shah, J., \& Higgins, E. T. (1997). Expectancy x value effects: Regulatory focus as determinant of magnitude and direction. Journal of Personality and Social Psychology, 73, 447-458.

Shepperd, J. A. (1993). Student derogation of the scholastic aptitude test: Biases in perceptions and presentations of college board scores. Basic and Applied Social Psychology, 14, 45-473.

Stajkovic, A. D., \& Luthans, F. (1997). A meta-analysis of the effects of organizational behavior modification on task performance. Academy of Management Journal, 41, 1122-1149.

Stocker, D., Jacobshagen, N., Annen, H., \& Semmer, N. K. (in press). Appreciation at work in the Swiss military forces. Swiss Journal of Psychology.

Tesser, A. (2001). On the plasticity of self-defense. Current Directions in Psychological Science, 10, 66-69.

Tourish, D., \& Robson, P. (2006). Sensemaking and the distortion of critical upward communication in organizations. Journal of Management Studies, 43, 711-730.

Trope, Y., \& Neter, E. (1994). Reconciling competing motives in self-evaluation: The role of selfcontrol in feedback-seeking. Journal of Personality and Social Psychology, 66, 646-657.

Ullrich, J., Christ, O., \& van Dick, R. (2009). Substitutes for procedural fairness: Prototypical leaders are endorsed whether they are fair or not. Journal of Applied Psychology, 94, 235-244.

Van Dijk, D. \& Kluger, A. N. (2004). Feedback sign effect on motivation: Is it moderated by regulatory focus? Applied Psychology: An International Review, 53, 113-135. 
Van-Dyk, C., Frese, M., Baer, M., \& Sonnentag, S. (2005). Organizational error management culture and its impact on performance: A two-study-replication. Journal of Applied Psychology, 90, 1228-1240.

Williams, K. D. (2007). Ostracism. Annual Review of Psychology, 58, 425-452.

Zohar, D. (2002), Modifying supervisory practices to improve subunit safety: A leadership-based intervention model. Journal of Applied Psychology, 87, 156-163.

Prof. Dr. Norbert K. Semmer ist Professor für Arbeits- und Organisationspsychologie am Institut für Psychologie der Universität Bern.

Dr. Nicola Jacobshagen ist Oberassistentin an der Abteilung Arbeits- und Organisationspsychologie am Institut für Psychologie der Universität Bern. 